Mimicker of Fitz-Hugh-Curtis syndrome:

\title{
A case report of perihepatitis secondary to sigmoid colonic perforation
}

Sirote Wongwaisayawan, M.D..$^{(1)}$

Pinporn Jenjitranant, M.D. ${ }^{(1)}$

Natthawut Jarunnarumol, M.D. ${ }^{(1)}$

Goragoch Gesprasert, M.D., Ph.D. ${ }^{(2)}$

Nitima Saksobhavivat, M.D. ${ }^{(1)}$

From ${ }^{(1)}$ Department of Diagnostic and Therapeutic Radiology, Faculty of Medicine Ramathibodi Hospital, Mahidol University, Bangkok, Thailand.

(2) Department of Surgery, Faculty of Medicine Ramathibodi Hospital, Mahidol University, Bangkok, Thailand.

Address correspondence to S.W. (e-mail: sirote.won@mahidol.edu)

Received 25 March 2021; revised 11 April 2021; accepted 12 April 2021

doi:10.46475/aseanjr.v22i1.123

\section{Abstract}

Perihepatitis is an uncommon condition defined as the inflammation of the peritoneal liver capsule. It is more commonly known as Fitz-Hugh-Curtis syndrome, a rare condition characterized by the inflammation of the peritoneum and tissue around the liver. This syndrome is usually associated with pelvic inflammatory disease (PID). However, PID is not always the cause of perihepatitis. We reported a rare case of perihepatitis which occurs secondarily to sigmoid colonic perforation.

Keywords: Perihepatitis syndrome, Sigmoid colon, Intestinal perforation, Fitz-Hugh-Curtis syndrome. 


\section{Introduction}

Perihepatitis is an uncommon condition that is defined as the inflammation of the peritoneal liver capsule. It is traditionally described as Fitz-Hugh-Curtis syndrome, a rare condition characterized by secondary inflammation of the peritoneum and tissue around the liver from pelvic inflammatory disease (PID) $[1,2]$. On a computed tomography (CT) scan, the inflammation of tissue around the liver may be seen as an intense enhancement of the liver capsule. This CT finding can serve as a hint to evaluate the pelvic inflammatory condition, particularly PID or tubo-ovarian abscess (TOA). However, PID or TOA is not always the cause of perihepatitis. This case report presents a rare case of perihepatitis which occurs secondarily to sigmoid colonic perforation.

\section{Case Summary}

An 88-year-old woman was presented to the emergency department (ED) with progressive epigastric pain for two days. She denied any nausea, vomiting, and fever. She had a past history of chronic deep venous thrombosis (DVT) of the left leg and a previous hysterectomy with bilateral oophorectomy.

On admission, the patient had a blood pressure of $143 / 76 \mathrm{~mm} \mathrm{Hg}$, a heart rate of $112 \mathrm{bpm}$, and a body temperature of 36.7 degrees Celcius. The physical examination revealed abdominal distension, decreased bowel sound, generalized guarding, and rebound tenderness in the epigastric area. The laboratory results showed a normal white blood cell count $\left(8.9 \times 10^{9} / \mathrm{L}\right)$ and an elevated serum lactate level of $5.2 \mathrm{mmol} / \mathrm{L}$ (normal range: $0.7-2.5 \mathrm{mmol} / \mathrm{L}$ ). The serum electrolyte panels and liver function tests were within normal limits.

An emergency contrast-enhanced CT scan at the ED revealed a small amount of perihepatic fluid and conspicuous intense enhancement of the liver capsule along the right hepatic lobe which represents perihepatitis (Figure 1). Segmental colitis with sigmoid colonic perforation, pneumoperitoneum, and 
fecal contamination in the peritoneal cavity are noted (Figure 2) and thought to be the causes of abdominal pain in this patient. Peritoneal fat inflammation in the right-sided abdomen is also seen. The patient underwent emergent exploratory laparotomy which revealed a $3-\mathrm{cm}$ sigmoid colonic perforation. Severe fecal contamination and severe adhesion of the entire abdomen were noted intraoperatively (Figure 3 ). The cause of sigmoid colonic perforation in this patient was presumed to be stercoral colitis. Adhesiolysis and Hartmann's procedure were performed. The patient tolerated the procedure without any immediate postoperative complications. The pathological result confirmed the diagnosis of sigmoid colonic perforation. No malignancy was found in the surgical specimen.

The patient made a satisfactory post-operative recovery in the intensive care unit (ICU) and was discharged from the hospital on postoperative day 23. She had been doing well at her last surgical outpatient follow-up.

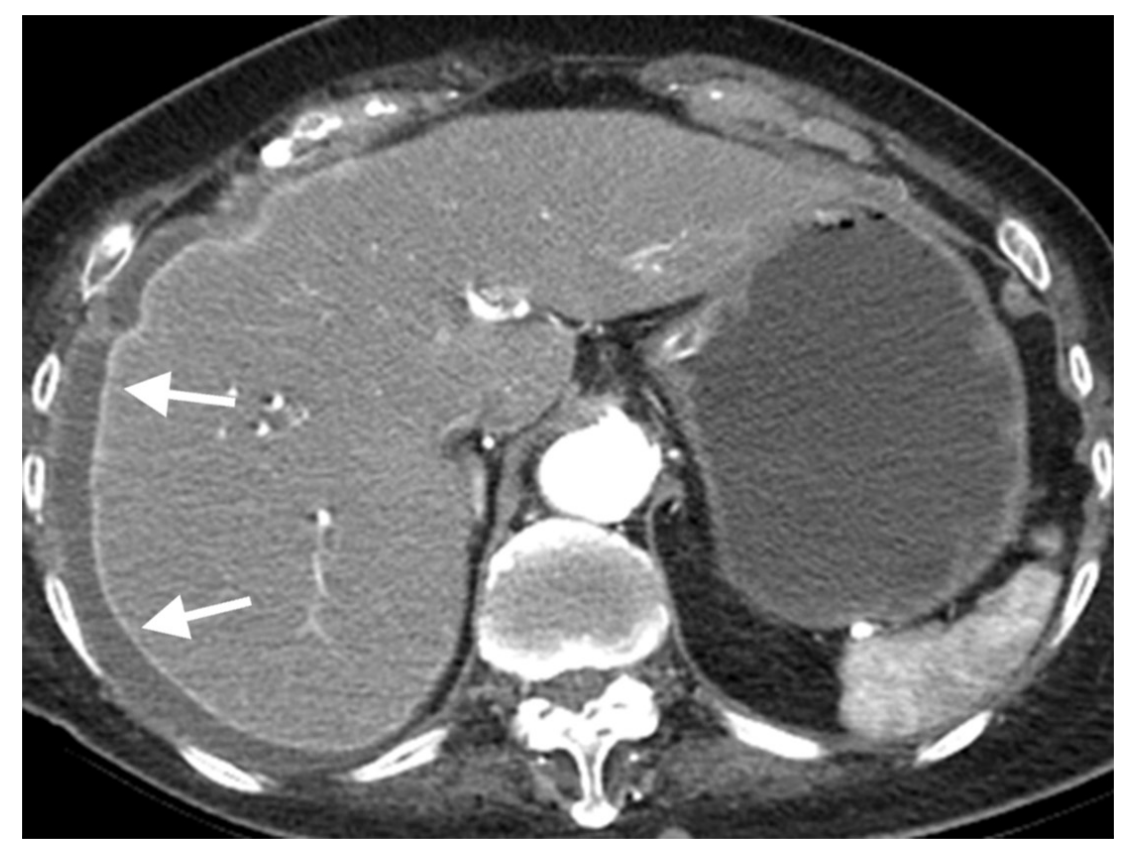

Figure 1. Axial contrast-enhanced computed tomography scan showing small amount of perihepatic fluid and intense enhancement of the liver capsule along the right hepatic lobe (arrows). 

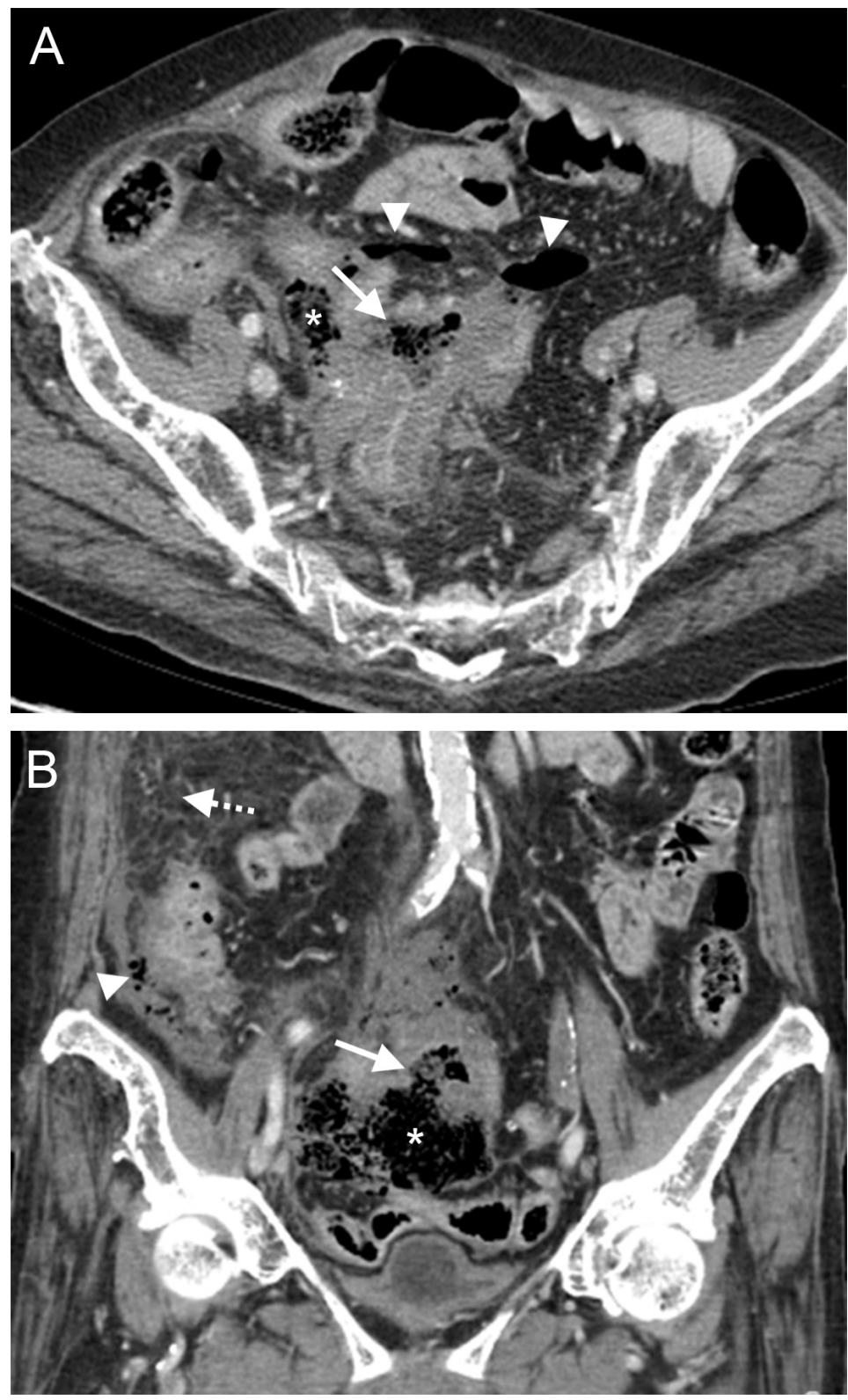

Figure 2. Contrast-enhanced computed tomography scan in axial (A) and coronal (B) views showing sigmoid colonic perforation (arrow) and fecal contamination in the peritoneal cavity (asterisk). Extraluminal free air in the pelvic cavity and right lower quadrant abdomen is observed (arrowheads). Peritoneal fat stranding along the right paracolic gutter is also seen (dashed arrow). 

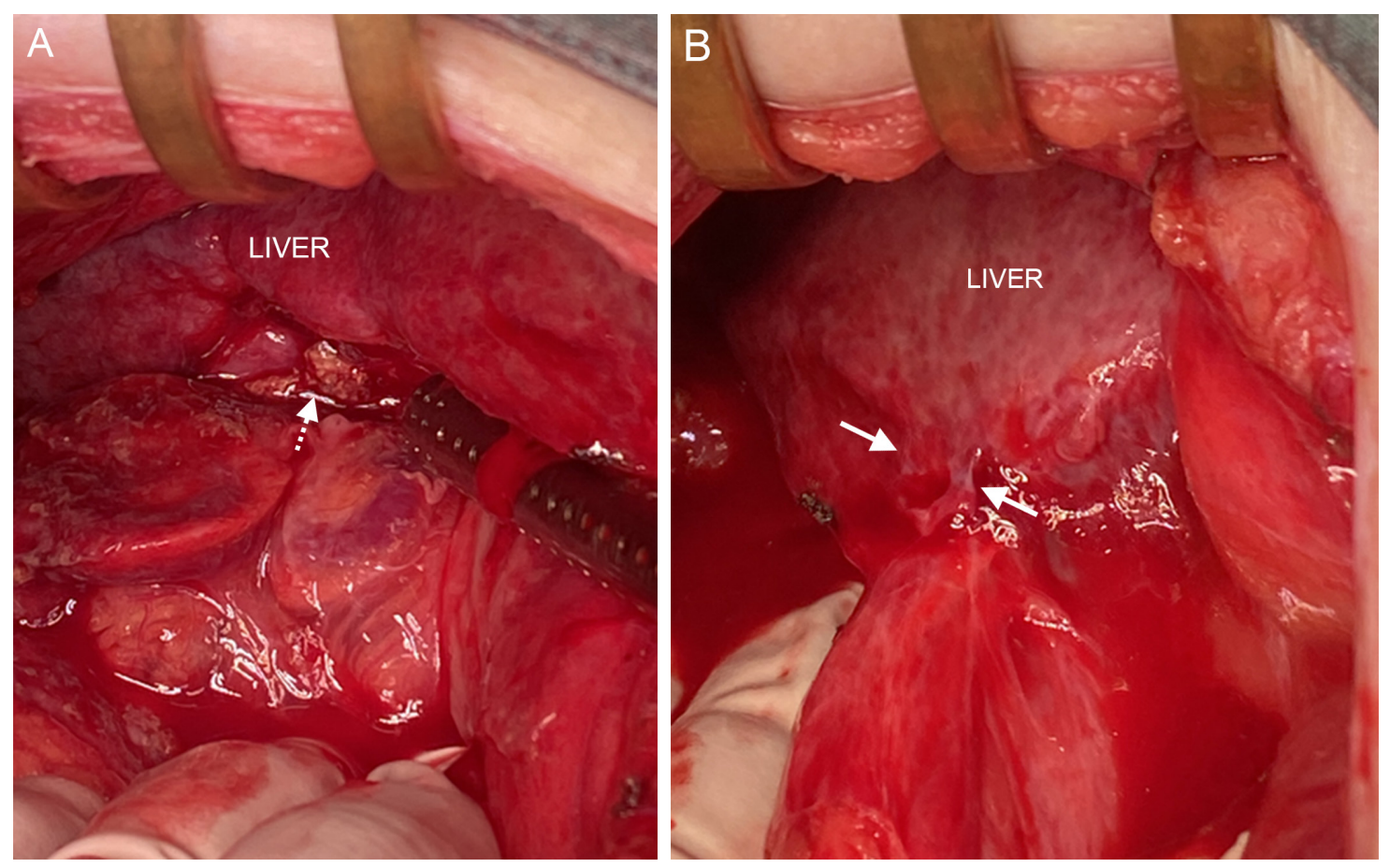

Figure 3. Intraoperative photographs $(A, B)$ showing fecal contamination (dashed arrow) and string-like adhesion (arrows) in the perihepatic space.

\section{Discussion}

Perihepatitis is defined as an inflammation of the liver capsule and tissue around the liver, regardless of the involvement of the liver parenchyma. It is classically described in association with the pelvic inflammatory disease (PID) caused by C. trachomatis or N. gonorrhoeae, the so-called "Fitz-Hugh-Curtis syndrome" $[1,2]$. Fitz-Hugh-Curtis syndrome is thought to be a result of the intraperitoneal spread of the infection from the pelvic cavity, with associated adhesion formation in the perihepatic region and the right upper quadrant (RUQ) abdomen [3]. The typical presented symptoms include RUQ abdominal pain and tenderness in a young reproductive female. The diagnosis of Fitz-Hugh-Curtis syndrome is usually confirmed by direct visualization of violin string-like adhesions through a laparoscopy or laparotomy, or by positive culture of C. trachomatis or $N$. gonorrhoeae in fluid from the pelvic cavity or in a cervical smear [3]. 
The inflammation and the increased blood flow of the peritoneum may manifest as intense enhancement of the liver capsule on an early-phase or arterial-phase CT image which is described as perihepatitis [4-6].

To our knowledge, this is the first case report of perihepatitis secondary to sigmoid colonic perforation, which mimics the CT findings of Fitz-HughCurtis syndrome. The pelvic inflammation in the present case was a result of the perforated sigmoid colon and intraperitoneal fecal contamination, rather than the classically described PID or TOA in Fitz-Hugh-Curtis syndrome. Although the presented patient did not have a confirmatory test for C. trachomatis or $N$. gonorrhoeae infection, the patient reported no active sexual activity and there were no suspicious intraoperative findings of PID or TOA. Moreover, the patient had undergone total hysterectomy many years before this presentation, making the occurrence of PID or TOA very unlikely.

Previous studies had reported $82 \%-86 \%$ overall accuracy of CT scan in predicting the site of bowel perforation $[7,8]$. In subtle cases, small loculated extraluminal air, pericolic fat stranding, and colonic wall thickening may be the only signs of the perforation site [9]. The presence of perihepatitis can increase the level of confidence in identifying the perforation site because the abnormality in the pelvic cavity is anticipated. The presence of extraluminal bowel content, loculated extraluminal air, bowel wall thickening, and defect of bowel wall in the pelvic cavity should be carefully assessed on CT images [7].

We postulate that the inflammatory fluid and fecal contamination in the pelvic cavity spread to the RUQ abdomen by means of transperitoneal ascitic flow. The peritoneal fluid in the pelvis is drawn to the upper abdomen, particularly at the right subdiaphragmatic region, secondary to the hydrostatic pressure variation from the diaphragmatic movement during inspiration [10]. Therefore, any pelvic inflammatory condition can theoretically result in the secondary inflammation of the perihepatic space or right subphrenic space. A similar finding of intense hepatic capsular enhancement has been reported in a patient with systemic lupus erythematosus [11]. Any condition that causes inflammation of the peritoneum 
of the RUQ abdomen may have intense enhancement of the liver capsule as well, such as cholecystitis, hepatic abscess, cholangitis, superior vena cava obstruction, tuberculous peritonitis, exposure of the liver to radiation, peritoneal carcinomatosis, and hepatic schistosomiasis [3]. However, other ancillary findings are usually present in most of these conditions and can serve as clues to the correct diagnosis.

\section{Conclusion}

We described a rare cause of perihepatitis resulting from sigmoid colonic perforation. The presence of perihepatitis on a CT scan should raise the possibility of the pelvic inflammatory condition. Therefore, the abnormality in the pelvic cavity should be carefully evaluated on a CT scan. The radiologists' awareness of this condition can help facilitate the correct diagnosis and guide the appropriate treatment. 


\section{References}

1. Fitz-Hugh T. Jr. Acute gonococcic peritonitis of the right upper quadrant in women. JAMA 1934;102:2094-6.

2. Curtis AH. A cause of adhesions in the right upper quadrant. JAMA 1930;94.1221-2.

3. Kim S, Kim TU, Lee JW, Lee TH, Lee SH, Jeon TY, et al. The perihepatic space: comprehensive anatomy and CT features of pathologic conditions. Radiographics 2007;27:129-43. doi: 10.1148/rg.271065050.

4. Tsubuku M, Hayashi S, Terahara A, Furukawa T, Ohmura G. Fitz-Hugh-Curtis syndrome: linear contrast enhancement of the surface of the liver on CT. J Comput Assist Tomogr 2002;26:456-8. doi: 10.1097/00004728-20020500000025 .

5. Nishie A, Yoshimitsu K, Irie H, Yoshitake T, Aibe H, Tajima T, et al. FitzHugh-Curtis syndrome. Radiologic manifestation. J Comput Assist Tomogr 2003;27:786-91. doi: 10.1097/00004728-200309000-00017.

6. Joo SH, Kim MJ, Lim JS, Kim JH, Kim KW. CT Diagnosis of Fitz-Hugh and Curtis Syndrome: value of the arterial phase scan. Korean J Radiol 2007;8:407. doi: 10.3348/kjr.2007.8.1.40.

7. Hainaux B, Agneessens E, Bertinotti R, De Maertelaer V, Rubesova E, Capelluto E, et al. Accuracy of MDCT in predicting site of gastrointestinal tract perforation. AJR Am J Roentgenol 2006;187:1179-83. doi: 10.2214/ AJR.05.1179.

8. Catalano O. [Computed tomography in the study of gastrointestinal perforation]. Radiol Med 1996;91:247-52. Italian. 
9. Singh JP, Steward MJ, Booth TC, Mukhtar H, Murray D. Evolution of imaging for abdominal perforation. Ann R Coll Surg Engl 2010;92:182-8. doi:10.1308/ $003588410 X 12664192075251$.

10. Pannu HK, Oliphant $M$. The subperitoneal space and peritoneal cavity: basic concepts. Abdom Imaging 2015;40:2710-22. doi: 10.1007/s00261-015-0429-5.

11. Schoenwaelder M, Stuckey SL. Perihepatitis associated with systemic lupus erythematosus: computed tomography findings. Australas Radiol 2005;49:17981. doi: 10.1111/j.1440-1673.2005.01370.x. 